\title{
Engineering band gap of bare titanium dioxides for visible-light activity: a theoretical prediction
}

\author{
Yun Wang, ${ }^{\text {a }}$ Haimin Zhang, ${ }^{a}$ Porun Liu,,${ }^{\text {X Xiangdong Yao, }}{ }^{\mathrm{b}}$ Huijun Zhao, ${ }^{\text {a }}$ \\ Received (in $X X X, X X X)$ Xth $X X X X X X X X X 20 X X$, Accepted $X$ th $X X X X X X X X X 20 X X$ \\ ${ }_{5}$ DOI: 10.1039/b000000x
}

The main obstacle for the practical applications of common titanium dioxide $\left(\mathrm{TiO}_{2}\right)$ photocatalysts is that they can only absorb ultraviolet sunlight. The fluorite $\mathrm{TiO}_{2}$ has attracted considerable interests since they may utilize more abundant visible sunlight. However, their electronic properties have not yet been confirmed according to the previous theoretical investigations. In this study, density functional theory

10 with different exchange-correlation functionals was employed to explore the electronic structures of fluorite $\mathrm{TiO}_{2}$ materials. Our results demonstrate that the prefect fluorite $\mathrm{TiO}_{2}$ crystals are indirect semiconductors. The size of supercells plays an important role for the calculation outcomes due to the Brillouin zone folding. The theoretical band gap energy of fluorite $\mathrm{TiO}_{2}$ using hybrid DFT method is 2.31 $\mathrm{eV}$, which support its photocatalytic activity over visible light. The surrounding static electric fields of Ti

15 cations of fluorite $\mathrm{TiO}_{2}$ are responsible to the reduced bang gap energies on the basis of crystal field theory. According to our theoretical deduction, possible visible-light responsive $\mathrm{TiO}_{2}$ photocatalysts were proposed by tuning the atomic configurations of the rutile $\mathrm{TiO}_{2}(110)$ surface.

\section{Introduction}

Titanium dioxide $\left(\mathrm{TiO}_{2}\right)$ photocatalysts have demonstrated 20 capabilities to directly utilize solar energy to remediate environment or generate clean energies. ${ }^{1-4}$ Although notable progress has been achieved in recent years, the wide band gap energies of the common $\mathrm{TiO}_{2}$ polymorphs (> $\left.3.0 \mathrm{eV}\right)$ limit their photoactivity to the ultraviolet (UV) region of the sunlight ${ }_{25}$ spectrum, which is just $5 \%$ of the sun-light. ${ }^{5-6}$ To tune $\mathrm{TiO}_{2}$ into visible-light responsive materials, various band gap engineering approaches have been developed through chemical modifications, such as cation or anion doping, ${ }^{7}$ alloying, ${ }^{8}$ sensitization by organic dye or inorganic quantum dots, and upconversion. ${ }^{3,}$ 9-11

30 These approaches have succeeded in reducing the band gap energies of $\mathrm{TiO}_{2}$ photocatalysts to realize visible-light photocatalytic activity, such as the nitrogen doped $\mathrm{TiO}_{2}$ materials. ${ }^{11-12}$ However, the energy conversion efficiencies of modified $\mathrm{TiO}_{2}$ photocatalysts are still too low for practical 35 applications. ${ }^{3}$ One of the disadvantages of these chemical modification approaches lies on the fact that the modifiers can be the recombination or trapping centres for the photo-generated charge carriers. ${ }^{9}$ Therefore, synthesizing visible-light active $\mathrm{TiO}_{2}$ photocatalysts without any chemical modifications becomes 40 attractive. ${ }^{13-15}$

$\mathrm{TiO}_{2}$ shows the rich phase diagram under wild or elevated pressures and temperatures, such as rutile, anatase, brookite, baddeleyite, fluorite, and cotunnite phases. ${ }^{16-17}$ The previous theoretical studies indicated that the pure fluorite-type $\mathrm{TiO}_{2}$ 45 crystals may be visible-light active. ${ }^{16,}{ }^{18-20}$ Among all the properties of fluorite $\mathrm{TiO}_{2}$, their electronic structures are the key feature to determine their performance in photocatalysis or solar power generation applications. Unfortunately, the direct detection of their electronic properties is rather difficult in experiments 50 since fluorite $\mathrm{TiO}_{2}$ materials can only be synthesized under high pressure $\left(>48 \mathrm{GPa}\right.$ ) around $2000 \mathrm{~K}^{21}$ To explore the electronic structures of the fluorite $\mathrm{TiO}_{2}$, some theoretical studies have been performed based on the traditional density functional theory (DFT), quasi-particle approximation (GW) or quantum Monte ${ }_{55}$ Carlo (QMC) method. ${ }^{16,18-20}$ However, the previous theoretical conclusions on their electronic properties are inconsistent. Kong et al., Mattesini et al., and Abbasnejad et al. found that the fluorite $\mathrm{TiO}_{2}$ was an indirect semiconductor according to their band structures using the DFT, GW or QMC methods. ${ }^{18-20}$ ${ }_{60}$ Conversely, Kuo et al. proposed that the fluorite $\mathrm{TiO}_{2}$ was a direct semiconductor. ${ }^{16}$ Since the electronic structures are crucial in determining the photoactivity of $\mathrm{TiO}_{2}$ materials, the precise understanding of their electronic properties is thoroughly necessary.

65 In the previous theoretical studies on the fluorite $\mathrm{TiO}_{2}$, the DFT method using local (local density approximation, LDA) or semi-local (general gradient approximation, GGA) exchange correlation (XC) functionals has been employed. ${ }^{16,}$ 19-20 These DFT calculations generally underestimate the band gap energies 70 of semiconductors to the extent of $30 \% .^{22-25}$ While the GW and QMC methods used in the previous studies are more advanced technologies to get better electronic structures of materials, the current computation resource still limits their applications. ${ }^{18-19,26}$ An alternative to deal with these issues in a first-principle manner 75 is the hybrid DFT method, in which a component of non-local Hartree-Fock exchange is included into the XC functional. ${ }^{27-28} \mathrm{In}$ 

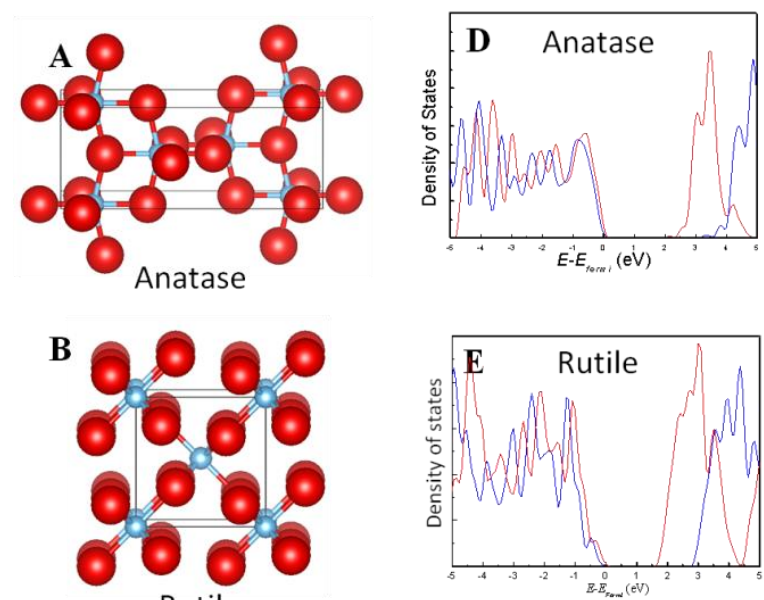

Rutile

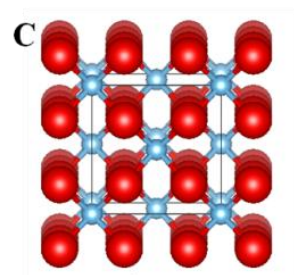

Fluorite

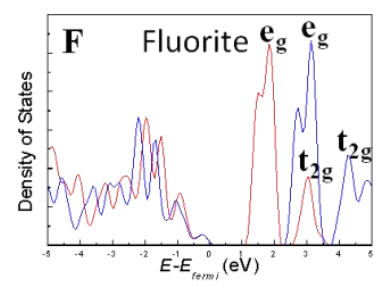

Fig. 1 Atomic structures of (A)anatase; (B)rutile; and (C) fluorite $\mathrm{TiO}_{2}$ crystals along with their total density of states (TDOS) of (D) anatase; (E) rutile; and (F) fluorite $\mathrm{TiO}_{2}$ crystals with the $\mathrm{PBE}$ (red) or HSE06 (blue) 5 functional. The black frames in (A-C) represent the unit cells of crystals. Key: red-oxygen, blue-titanium.

this work, we employed the recently developed screened hybrid functionals in periodic systems to examine the electronic structures of fluorite $\mathrm{TiO}_{2}$ materials. ${ }^{27}$ Our calculations 10 demonstrate that they are indirect semiconductors with the visible-light activity. The orientation of the $\mathrm{Ti} 3 d$ orbitals of fluorite $\mathrm{TiO}_{2}$ in their surrounding static electric fields plays the key role to reduce their band gap energy.

\section{Computation Methods}

15 All calculations were performed using the Vienna $a b$ initio simulation program (VASP) based on the DFT with the allelectron projected augmented wave (PAW) method in this study. ${ }^{29-31}$ A plane-wave basis set was employed with a kinetic energy cut-off of $400 \mathrm{eV}$. Valence states included the Ti $3 p, 4 s$, 20 and $3 d$ states, and the $\mathrm{O} 2 s$ and $2 p$ states. We use the $\mathrm{PBE}^{32}$ and HSE06 XC functional ${ }^{33}$ for the GGA-level and screened hybrid DFT-level calculations, respectively. The screening parameter for exchange of the HSE06 functional was $0.2 \AA^{-1}$. ${ }^{4}$ An exchange functional of $20 \%$ exact $\mathrm{HF}$ exchange with $80 \%$ PBE exchange

25 was employed in this study since calculations with such ratio accurately predicted the lattice constants and the band gap for rutile and anatase $\mathrm{TiO}_{2}$ materials. ${ }^{35-36}$

To understand the origin of the visible-light activity of fluorite $\mathrm{TiO}_{2}$, the structural and electronic properties of rutile, anatase and 30 fluorite $\mathrm{TiO}_{2}$ polymorphs were calculated and compared. We performed Brillouin-zone (BZ) integrations using Monkhorst-
Table 1 Theoretical and experimental structural parameters and their band gap energy $(\mathrm{Eg})$ for anatase, rutile and fluorite $\mathrm{TiO} 2$.

\begin{tabular}{cccccc}
\hline Method & $\mathrm{a}(\AA)$ & $\mathrm{b}(\AA)$ & $\mathrm{c}(\AA)$ & $\mathrm{u}$ & $E_{g}(\mathrm{eV})$ \\
\hline & & & & anatase & \\
PBE & 3.801 & 3.801 & 9.711 & 0.207 & 2.143 \\
HSE06 & 3.770 & 3.770 & 9.626 & 0.206 & 3.341 \\
exp. $^{37}$ & 3.782 & 3.782 & 9.502 & 0.208 & $3.2-3.4$ \\
& & & & rutile & \\
PBE & 4.641 & 4.641 & 2.970 & 0.305 & 1.802 \\
HSE06 & 4.593 & 4.593 & 2.953 & 0.305 & 3.083 \\
exp. $^{37}$ & 4.593 & 4.593 & 2.959 & 0.305 & 3.0 \\
& & & & fluorite & \\
PBE & 4.833 & 4.833 & 4.833 & & 1.099 \\
HSE06 & 4.786 & 4.786 & 4.786 & & 2.306 \\
exp. $^{21}$ & 4.870 & 4.870 & 4.870 & & \\
\hline
\end{tabular}

${ }_{35}$ Pack grids of special points with $(4 \times 4 \times 6),(6 \times 6 \times 2)$ and $(6 \times 6 \times 6)$ meshes for rutile, anatase and fluorite $\mathrm{TiO}_{2}$ supercells, respectively. Each supercell of rutile $\mathrm{TiO}_{2}$ includes 6 atoms while each anatase or fluorite supercell has 12 atoms, as shown in the black frames of Fig. 1A-C. The rutile (110) surface atoms were 40 engineered to form fluorite-type structures in this study because this surface is the most well studied one. The rutile (110) surface was modelled by a slab with the $(1 \times 1)$ surface cell comprising an 18 atomic layers separated by a vacuum region of $10 \AA$. A $(4 \times 6 \times 1) \mathrm{k}$-point mesh was used in this study. The reconstruction 45 occurs in one surface of the slabs. When the geometry was optimized, all the atoms were allowed to relax. The corresponding k-mesh densities and the cut-off kinetic energy have been justified in our previous studies. ${ }^{38-40}$ The other calculation parameters for the hybrid DFT calculations have been

50 validated below through the comparison with the experimental values.

\section{Results and discussion}

\subsection{Rutile and Anatase}

To validate the calculation parameters for the hybrid DFT 55 calculations in this study, we have calculated the structural and electronic properties of rutile and anatase $\mathrm{TiO}_{2}$ crystals firstly. Rutile and anatase are the most common polymorphs of $\mathrm{TiO}_{2}$ in nature and practical applications. The theoretical lattice constants and band gap energies are listed in Table 1, along with the 60 experimental data. ${ }^{16,37}$ It can be found that the theoretical lattice constants calculated both with the PBE and HSE06 functionals are very close to the experimental values. The biggest discrepancy is less than $0.1 \AA$. However, the band gap energies of rutile and anatase materials using the PBE functional are about ${ }_{65} 40 \%$ lower than the corresponding experimental values $(3.00 \sim$ $3.40 \mathrm{eV}) .{ }^{41-44}$ Using the HSE06 XC functional, the theoretical bang gap energies are 3.08 and $3.34 \mathrm{eV}$ for the rutile and anatase $\mathrm{TiO}_{2}$, respectively. The significant improvement through the hybrid DFT calculations confirms the advantage of this method 70 employed in this study. The images of density of states (DOS) of rutile and anatase $\mathrm{TiO}_{2}$ materials are also shown in Fig. 1D-E, which will be used to be compared with that of the fluorite $\mathrm{TiO}_{2}$. Our results are similar as those using the same level computational methods, ${ }^{35-36,} 45$ which demonstrates that the 75 calculation parameters used in this study are valid.

\subsection{Structural and electronic properties of fluorite $\mathrm{TiO} 2$}




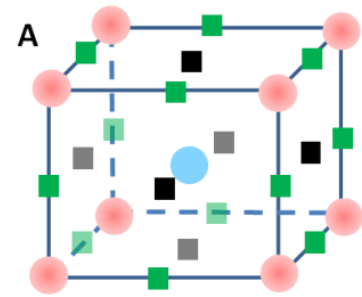

Cubic $\left[\mathrm{TiO}_{8}\right]$

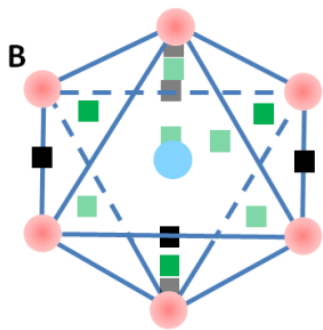

Octahedral $\left[\mathrm{TiO}_{6}\right]$
Fig. 2 Illustrations of (A) the cubic $\left[\mathrm{TiO}_{8}\right]$ in fluorite $\mathrm{TiO}_{2}$ and (B) octahedral $\left[\mathrm{TiO}_{6}\right]$ in rutile or anatase $\mathrm{TiO}_{2}$. Key: red-oxygen, bluetitanium, black-orientation of Ti $3 d \mathrm{t}_{2 \mathrm{~g}}$ orbitals, and green-orientation of Ti $3 d \mathrm{e}_{\mathrm{g}}$ orbitals.

5 Compared with rutile and anatase $\mathrm{TiO}_{2}$, the fluorite-type crystals show higher symmetry. The atomic structure of fluorite $\mathrm{TiO}_{2}$ crystals belongs to the cubic $F m 3 m$ space group, as shown in Fig. $1 \mathrm{C}^{21}$ After the structure optimization, the theoretical lattice constants are 4.833 and $4.786 \AA$ for PBE and HSE06 10 functional, respectively (see Table 1). Our results are in agreement with to the previous theoretical values, but slightly less than the experimental data. ${ }^{16,18-21}$ It should be noted that the experimental data was not directly measured since the fluorite $\mathrm{TiO}_{2}$ can only be stable at the high pressure and high 15 temperature. ${ }^{21}$ The experimental lattice constant was extrapolated according to the lattice constants measured under 10-55 Gpa pressure. ${ }^{21}$ Thus, the theory-experiment discrepancy may be not fully ascribed to the computation accuracy. According to our PBE results, the average volume per $\mathrm{TiO}_{2}$ unit in fluorite crystals is $2028.2 \AA^{3}$, which is at least $13.3 \%$ smaller than those in rutile and anatase crystals. It matches the experiments that the fluorite phase can only be produced under the high pressure. ${ }^{21}$

From Table 1, the theoretical band gap energy with PBE functional is $1.10 \mathrm{eV}$, which is $1.21 \mathrm{eV}$ lower than that with ${ }_{25}$ HSE06 functional. Our PBE value is close to the previous ones obtained using the same level functionals. ${ }^{16,20}$ And the band gap energy obtained through the screened hybrid DFT method is similar to that using GW $(2.37 \mathrm{eV})^{19}$ or QMC $(2.44 \mathrm{eV})^{18}$ methods. Since the computation costs of the screened hybrid DFT 30 method are much less than that by using GW and QMC methods, the great advantage of the screened hybrid DFT method used in this study is shown.

On the basis of our calculations, the band gap energy of fluorite $\mathrm{TiO}_{2}$ is about $1.0 \mathrm{eV}$ lower than those of rutile and 35 anatase phases. The reduced band gap energy of fluorite $\mathrm{TiO}_{2}$ is mainly due to the change of the surrounding chemical environments of Ti atoms. ${ }^{16}$ In rutile and anatase $\mathrm{TiO}_{2}$ crystals, the coordination number $(\mathrm{CN})$ of $\mathrm{Ti}$ atoms is six through the formation of $\left[\mathrm{TiO}_{6}\right]$ octahedra with the $D_{2 h}$ symmetry. In fluorite 40 crystals, the $\mathrm{CN}$ of $\mathrm{Ti}$ atoms is eight in the perfect $\left[\mathrm{TiO}_{8}\right]$ cubes with the $O_{h}$ symmetry (see Fig. 2). According to the crystal field theory, the five degenerate Ti $3 d$ orbitals split into $t_{2 g}$ and $e_{g}$ orbitals by the interactions between the Ti $3 d$ electrons and their static electric fields generated by the surrounding $\mathrm{O}$ anions. In the ${ }_{45}\left[\mathrm{TiO}_{8}\right]$ cubes, the Ti $3 d \mathrm{t}_{2 \mathrm{~g}}$ orbitals point to the edge of the cubes (the green squares in Fig. 2A). And the Ti $3 d \mathrm{e}_{\mathrm{g}}$ orbitals points to the face centres of the cubes (the black squares in Fig. 2A). As a result, the shorter distances from Ti $3 d$ eg orbitals to the oxygen atoms $\left(0.5 d_{\mathrm{O}-\mathrm{O}}\right.$, and the $d_{\mathrm{O}-\mathrm{O}}$ is the shortest $\mathrm{O}-\mathrm{O}$ distance in
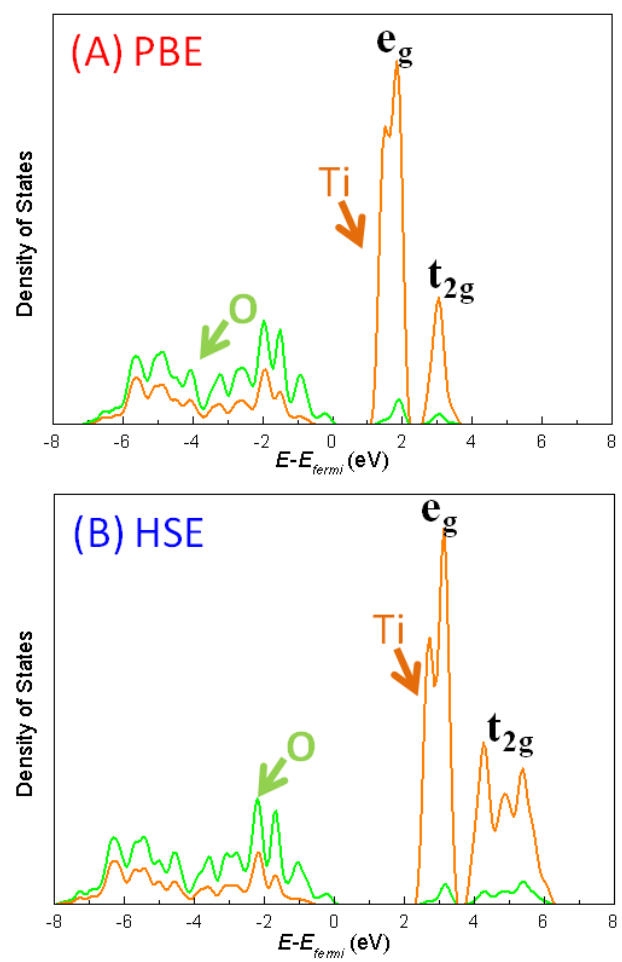

50 Fig. 3 Partial density of states (PDOS) of Ti (orange lines) and O (green lines) atoms in fluorite $\mathrm{TiO}_{2}$ with the (A) PBE or (B) HSE06 functional

crystals.) lead to the stronger repulsion between the electrons in Ti $3 d \mathrm{t}_{2 \mathrm{~g}}$ orbitals and the surrounding $\mathrm{O}$ anions. Such repulsion 55 leads that the energy levels of Ti $3 d t_{2 g}$ orbitals are lower than those of $e_{g}$ orbitals. ${ }^{16,20}$ The partial DOS (PDOS) images of Ti and $\mathrm{O}$ atoms in fluorite $\mathrm{TiO}_{2}$ have been calculated with different functionals, as shown in Fig. 3. The PDOS images confirm the order of conduction bands in fluorite $\mathrm{TiO}_{2}$ according to the ${ }_{60}$ deduction of the crystal field theory.

On the other hand, the symmetry of the $\left[\mathrm{TiO}_{6}\right]$ octahedra in rutile and anatase $\mathrm{TiO}_{2}$ is different from that of the $\left[\mathrm{TiO}_{8}\right]$ cubes. The distances from the Ti $3 d$ eg orbitals in the $\left[\mathrm{TiO}_{6}\right]$ octahedra are shorter since they points to the edge of the $\left[\mathrm{TiO}_{6}\right]$ octahedra ${ }_{65}$ (the black squares in Fig. 2B). Thus, the energy levels of Ti $3 d \mathrm{t}_{2 \mathrm{~g}}$ bands in the rutile and anatase $\mathrm{TiO}_{2}$ are lower than those of $\mathrm{e}_{\mathrm{g}}$ bands. Such electronic structure is reverse to that in the fluorite $\mathrm{TiO}_{2}{ }^{16,20}$ In addition, the distance from the Ti $3 d \mathrm{t}_{2 \mathrm{~g}}$ orbitals to $\mathrm{O}$ anions is $0.577 d_{\mathrm{O}-\mathrm{O}}$ in the $\left[\mathrm{TiO}_{6}\right]$ octahedra, which is shorter than 70 that in the $\left[\mathrm{TiO}_{8}\right]$ cubes $\left(0.707 d_{\mathrm{O}-\mathrm{O}}\right)$. As a result, the Ti $3 d \mathrm{e}_{\mathrm{g}}$ bands in the $\left[\mathrm{TiO}_{8}\right]$ cubes shift towards the Fermi energy level because of the relatively weaker repulsion between the electrons in Ti $3 d \mathrm{e}_{\mathrm{g}}$ and $\mathrm{O}$ anions. (see Fig. 1). The band gap energy of fluorite $\mathrm{TiO}_{2}$, therefore, reduces. The theoretical band gap energy $75(2.31 \mathrm{eV})$ of the fluorite $\mathrm{TiO}_{2}$ supports that they can absorb visible sunlight with wavelength less than $537 \mathrm{~nm}$.

\subsection{Comparison between different functionals}

The data in Table 1 show that the calculations both with the PBE and HSE06 functionals can give accurate structural 80 properties of $\mathrm{TiO}_{2}$ materials. The crucial improvement using the screened hybrid DFT method is the band gap energies of systems. 

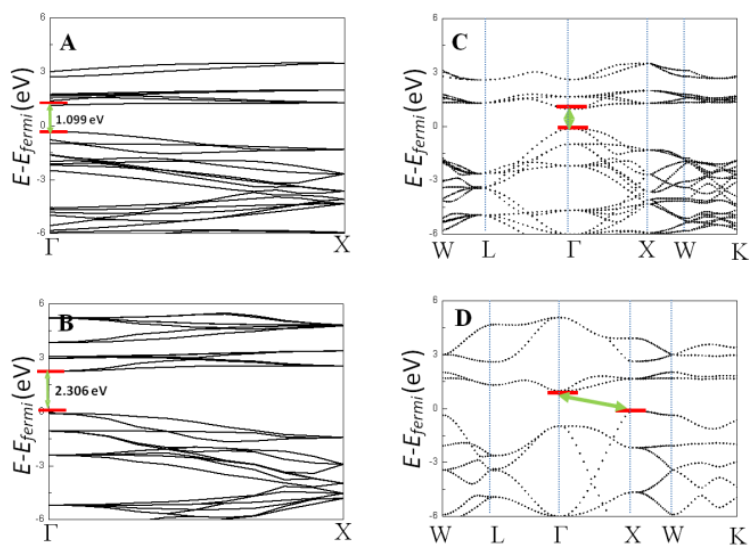

Fig. 4 Band structures of fluorite $\mathrm{TiO}_{2}$ crystals from $\Gamma$ to $\mathrm{X}$ symmetric points with (A) PBE and (B) HSE06 functional. And band structures from W-L- $\Gamma-\mathrm{X}-\mathrm{W}-\mathrm{K}$ of fluorite $\mathrm{TiO}_{2}$ crystals with $(\mathrm{C})$ a conventional unit cell including four $\mathrm{Ti}$ atoms and eight $\mathrm{O}$ atoms, or (D) a primitive cell (it 5 includes one $\mathrm{Ti}$ atom and two $\mathrm{O}$ atoms) using the PBE functional.

Comparing the DOS images in Fig. 1 and 3, it can be found that the position of the conduction bands by using the GGA/DFT is about $1.0 \mathrm{eV}$ lower than that and screened hybrid DFT methods, which leads to the underestimation of the band gap energies of all $10 \mathrm{TiO}_{2}$ materials. This discrepant value $(\sim 1.0 \mathrm{eV})$ has been used in GGA/DFT calculations to adjust the band gap energies while it is totally empirical. ${ }^{16}$ Another differentia related to the functionals is the width of the bands. According to our calculations, the widths of bands with the PBE functional are smaller than the 15 counterparts with HSE06 functional. One example is that the width of upper valence band is $7.4 \mathrm{eV}$ with PBE functional for the fluorite $\mathrm{TiO}_{2}$, which is about $0.7 \mathrm{eV}$ smaller than that with HSE06 functional. From Fig. 3 , the Ti $3 d \mathrm{t}_{2 \mathrm{~g}}$ band width with the HSE06 functional is almost twice as that using the PBE 20 functional. However, the DOS images in Fig. 1 and 3 also indicate that the order and shapes of the various bands of $\mathrm{TiO}_{2}$ are similar. Such similarities suggest that we may still use the GGA/DFT methods, which can reduce computation costs significantly to study more complicated systems, to 25 approximately analyze the electronic properties of $\mathrm{TiO}_{2}$ materials.

\subsection{Nature of electronic structure}

The main discrepancy of previous theoretical studies is whether fluorite $\mathrm{TiO}_{2}$ materials are direct semiconductors or not. Kong et al., Mattesini et al. and Abbasnejad et al. have proposed 30 that the fluorite $\mathrm{TiO}_{2}$ is an indirect semiconductor. The indirect transition is from the conduction band minimum $(\mathrm{CBM})$ at the $\Gamma$ point to the valence band maximum (VBM) at $\mathrm{X}$ as the minimum band gap. ${ }^{18-20}$ On the contrary, Kuo et al. found that fluorite $\mathrm{TiO}_{2}$ is a direct semiconductor with the minimum band gap at the $\Gamma$ 35 symmetry point based on their GGA/DFT calculations. ${ }^{16}$ Since the hybrid DFT calculation can improve the prediction of band gap energies as those using the GW and QMC methods, the calculations using the hybrid DFT calculations are expected to repeat the conclusion using the GW or QMC methods. In this 40 regard, we have calculated the band structure of fluorite $\mathrm{TiO}_{2}$ from the $\Gamma$ point to the $\mathrm{X}$ point using both traditional DFT and screened hybrid DFT approaches, as shown in Fig. 4A-B.
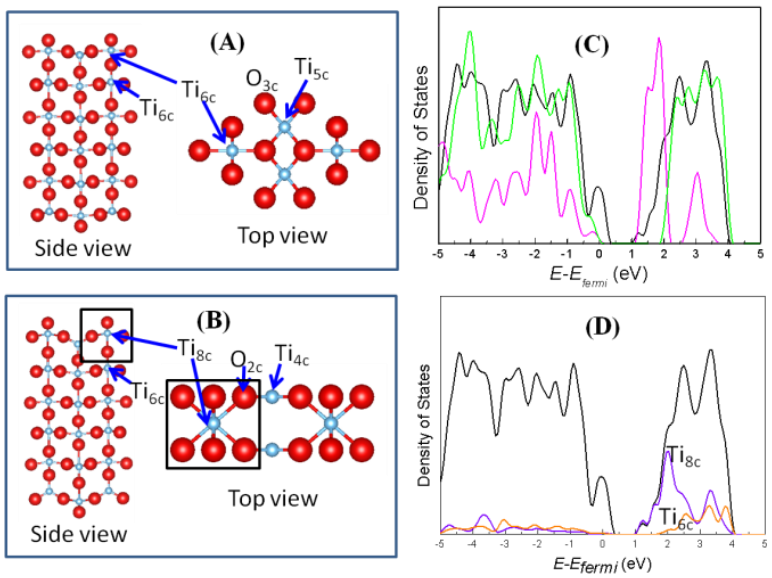

Fig. 5 (A) Atomic structure of regular rutile (110) surface; (B) Atomic structure of the reconstructed rutile (110) surface with $\left[\mathrm{TiO}_{8}\right]$ octahetra 45 (shown in the black rectangular frame); (C) TDOS of the fluorite $\mathrm{TiO}_{2}$ crystal (purple line), and the regular rutile (110) surface (green line) and the reconstructed rutile (110) surface (black line); and (D) TDOS of the reconstructed rutile (110) surface (black line), PDOS of eight-coordinated $\mathrm{Ti}$ atoms (magenta line) and six-coordinated $\mathrm{Ti}$ atoms in the bulk (orange 50 line). Key: red-oxygen, blue-titanium.

Unfortunately, the results suggest that the nature of band structures is independent to the choices of the functionals. The fluorite $\mathrm{TiO}_{2}$ is a direct semiconductor with the minimum band gap at the $\Gamma$ symmetry point both with the PBE and HSE06 55 functionals. There must be another factor causing the discrepancy found in the previous studies.

The previous studies on the anatase $\mathrm{TiO}_{2}$ revealed that the size of supercells in calculations may affect the band structures. ${ }^{45}$ Considering the computational costs of the GW and ${ }_{60} \mathrm{QMC}$ methods are very high, the primitive cell of the fluorite $\mathrm{TiO}_{2}$ crystal may be used in those calculations. The primitive cell of the fluorite $\mathrm{TiO}_{2}$ includes one $\mathrm{Ti}$ atom and two $\mathrm{O}$ atoms, which size is one quarter of that of the conventional unit cell used in this study before. Based on this consideration, the band structures of 65 the systems with primitive or conventional supercells were calculated using the PBE functional (see Fig. 4C-D). When the primitive cell was employed, the band structure shows that the fluorite $\mathrm{TiO}_{2}$ is an indirect semiconductor, which is same as those reported by Kong et al., Mattesini et al. and Abbasnejad et al.. ${ }^{18-}$

${ }^{70}{ }^{20}$ However, the system changes into a direct semiconductor when the bigger conventional supercell including four $\mathrm{Ti}$ atoms and eight $\mathrm{O}$ atoms was used. Thus, the size of supercells affects the band structures of fluorite $\mathrm{TiO}_{2}$. The size effect can be ascribed to the folding of the BZ. ${ }^{46}$ Since the conventional supercell is larger 75 than the primitive one, the first BZ of the conventional supercell is smaller. Consequently, the VBM at the $\mathrm{X}$ point in the first BZ of the primitive supercell is folded back onto the $\Gamma$ symmetry point in the first $\mathrm{BZ}$ of the conventional supercell, which causes the nature of the band structure of fluorite $\mathrm{TiO}_{2}$ materials 80 changes. Since the band structures using the primitive supercell describe the correct electronic properties of systems, the fluorite $\mathrm{TiO}_{2}$ are, therefore, demonstrated to be an indirect semiconductor.

\subsection{Reconstructed surfaces with $\left[\mathrm{TiO}_{8}\right]$}

85 Since the change of the static electric fields of Ti cations can 
tune the band gap of $\mathrm{TiO}_{2}$ into visible-light activity, engineering the surface structures to form highly symmetric $\left[\mathrm{TiO}_{8}\right]$ becomes a possible approach to avoid the high pressure required by the formation of the fluorite $\mathrm{TiO}_{2}$ bulk. To verify this theoretical 5 deduction, the stable rutile $\mathrm{TiO}_{2}$ (110) surface was deliberately reconstructed to form $\left[\mathrm{TiO}_{8}\right]$ in the surface. Two threecoordinated $\mathrm{O}$ atoms in the rutile $\mathrm{TiO}_{2}$ (110) surface were shifted half of unit vector length along $<001>$ direction to generate $\left[\mathrm{TiO}_{8}\right]$ cubes, as shown in Fig. 5A-B. After the atomic 10 optimization, the $\left[\mathrm{TiO}_{8}\right]$ in the surface are not with perfect $O_{h}$ symmetry, but very close to it. Such atomic structure can lead to the same electronic structures as that of the fluorite $\mathrm{TiO}_{2}$ since the static electric fields of Ti cations in both systems are similar. As a result, the similar visible-light activity can be expected.

15 The total DOS (TDOS) images shown in Fig. 5C demonstrate that the CBM of the reconstructed surface indeed shift towards the Fermi energy level. The location of CBM is close to that of the fluorite $\mathrm{TiO}_{2}$ crystal. The further PDOS analysis of the Ti atoms in the reconstructed surface (see the magenta line in Fig. 20 5D) reveals that the shift of the CBM is ascribed to the formation of the eight-coordinated surface $\mathrm{Ti}$ atoms in $\left[\mathrm{TiO}_{8}\right]$. As a comparison, the CBM of PDOS of six-coordinated Ti atoms in the bulk (the orange line in Fig. 5D) locates at the similar position of those of bulk rutile. The shift of CBM of the reconstructed 25 surface demonstrates that the visible-light response of the bare $\mathrm{TiO}_{2}$ photocatalysts can be achieved through the surface engineering to change the static electric fields of Ti cations in the surface. Recently, Etacheri et al. found that the oxygen-rich anatase $\mathrm{TiO}_{2}$ surfaces possessed visible-light activity. ${ }^{14}$ At the 30 same time, the reconstructed rutile (011) surface under the oxidizing condition was also found to be visible-light active without any doping. ${ }^{13}$ Such uncommon visible-light response may also be explained the formation of $\left[\mathrm{TiO}_{8}\right]$ cubes under the oxygen-rich conditions. The recent experiments to use the 35 hydrofluoric acid as a facet controlling agent to produce highenergy anatase (001) surface can be used as the model to synthesize visible-light active surfaces with $\left[\mathrm{TiO}_{8}\right]$ cubes. ${ }^{40,47-48}$

\section{Conclusions}

In this study, we have studies the rutile, anatase and fluorite $\mathrm{TiO}_{2}$ 40 crystals using both the GGA/DFT and the screened hybrid DFT methods. The employment of the screened hybrid DFT methods improves the prediction of the band gap energies of $\mathrm{TiO}_{2}$ photocatalysts significantly. The theoretical values with the HSE06 functional are almost identical to the experimental values.

45 Through our calculations, the fluorite $\mathrm{TiO}_{2}$ materials have been confirmed to be indirect semiconductors with minimum band gap from the CBM at the $\Gamma$ point to the VBM at the $\mathrm{X}$ point as the minimum band gap. The band gap energy as low as $2.31 \mathrm{eV}$ supports that the fluorite $\mathrm{TiO}_{2}$ phase is visible-light active. Such 50 visible-light response is related to the structure change of $\mathrm{TiO}_{2}$ by the formation of $\left[\mathrm{TiO}_{8}\right]$ cubes. The change of the static electric fields of Ti cations can tune the band gap energies of $\mathrm{TiO}_{2}$ on the basis of the crystal field theory. To this end, the $\mathrm{TiO}_{2}$ photocatalysts with $\left[\mathrm{TiO}_{8}\right]$ cubes in surfaces were proposed to be 55 visible-light active, which were theoretically confirmed in this study. Thus, surface engineering of $\mathrm{TiO}_{2}$ is a doable approach to achieve their visible-light activity without any doping.

\section{Acknowledgement}

We thank the Australian Research Council for funding this 60 work. This research was undertaken on the National Computation Infrastructure (NCI) in Canberra, Australia, which is supported by the Australian Commonwealth Government.

\section{Notes and references}

${ }^{a}$ Centre for Clean Environment and Energy, and Griffith School of 65 Environment, Griffith University, Gold Coast, QLD 4222, Australia Fax: +61-7-55528261; Tel: +61-7-55528261; E-mail: h.zhao@griffith.edu.au ${ }^{b}$ QLD Micro- and Nanotechnology Centre, Nathan Campus, Griffith University, QLD 4111, Australia

70 1. A. Fujishima and K. Honda, Nature, 1972, 238, 37-38.

2. B. Oregan and M. Gratzel, Nature, 1991, 353, 737-740.

3. R. M. N. Yerga, M. C. A. Galvan, F. del Valle, J. A. V. de la Mano and J. L. G. Fierro, ChemSusChem, 2009, 2, 471-485.

4. M. Gratzel, Accounts Chem. Res., 1981, 14, 376-384.

75 5. A. Fujishima and X. T. Zhang, C. R. Chim., 2006, 9, 750-760.

6. A. Fujishima, X. T. Zhang and D. A. Tryk, Surf. Sci. Rep., 2008, 63, 515-582.

7. W. J. Yin, H. W. Tang, S. H. Wei, M. M. Al-Jassim, J. Turner and Y. F. Yan, Phys. Rev. B, 2010, 82, 045106-045111.

80 8. Y. Q. Wang, X. D. Jiang and C. X. Pan, J. Alloy. Compd., 2012, 538, 16-20.

9. M. A. Henderson, Surf. Sci. Rep., 2011, 66, 185-297.

10. G. Liu, L. Z. Wang, H. G. Yang, H. M. Cheng and G. Q. Lu, J. Mater. Chem., 2010, 20, 831-843.

85 11. R. Asahi, T. Morikawa, T. Ohwaki, K. Aoki and Y. Taga, Science, 2001, 293, 269-271.

12. S. Livraghi, M. C. Paganini, E. Giamello, A. Selloni, C. Di Valentin and G. Pacchioni, J. Am. Chem. Soc., 2006, 128, 15666-15671.

13. J. G. Tao, T. Luttrell and M. Batzill, Nat. Chem., 2011, 3, 296-300.

90 14. V. Etacheri, M. K. Seery, S. J. Hinder and S. C. Pillai, Adv. Funct. Mater., 2011, 21, 3744-3752.

15. Y. H. Lu, B. Xu, A. H. Zhang, M. Yang and Y. P. Feng, J. Phys. Chem. C, 2011, 115, 18042-18045.

16. M. Y. Kuo, C. L. Chen, C. Y. Hua, H. C. Yang and P. Y. Shen, J. Phys. Chem. B, 2005, 109, 8693-8700.

17. J. Muscat, V. Swamy and N. M. Harrison, Phys. Rev. B, 2002, 65, 224112 .

18. M. Abbasnejad, E. Shojaee, M. R. Mohammadizadeh, M. Alaei and R. Maezono, Appl. Phys. Lett., 2012, 100, 261902.

100 19. X. G. Kong, Y. Yu and T. Gao, Eur. Phys. J. B, 2010, 76, 365-371.

20. M. Mattesini, J. S. de Almeida, L. Dubrovinsky, N. Dubrovinskaia, B. Johansson and R. Ahuja, Phys. Rev. B, 2004, 70, 115101.

21. M. Mattesini, J. S. de Almeida, L. Dubrovinsky, N. Dubrovinskaia, B. Johansson and R. Ahuja, Phys. Rev. B, 2004, 70, 212101.

105 22. J. Heyd, G. E. Scuseria and M. Ernzerhof, J. Chem. Phys., 2003, 118, 8207-8215.

23. J. Hafner, C. Wolverton and G. Ceder, MRS Bull., 2006, 31, 659-665.

24. Y. Zhao, J. Z. Pu, B. J. Lynch and D. G. Truhlar, Phys. Chem. Chem. Phys., 2004, 6, 673-676.

110 25. E. Finazzi, C. Di Valentin, G. Pacchioni and A. Selloni, J. Chem. Phys., 2008, 129, 154113.

26. H. Jiang, Acta Phys.-Chim. Sin., 2010, 26, 1017-1033. 
27. M. Marsman, J. Paier, A. Stroppa and G. Kresse, J. Phys.-Condes. Matter, 2008, 20, 064201.

28. Y. Wang, S. de Gironcoli, N. S. Hush and J. R. Reimers, J. Am. Chem. Soc., 2007, 129, 10402-10407.

5 29. G. Kresse and J. Hafner, Phys. Rev. B, 1993, 47, 558-561.

30. G. Kresse and J. Furthmuller, Comput. Mater. Sci., 1996, 6, 15-50.

31. G. Kresse and D. Joubert, Phys. Rev. B, 1999, 59, 1758-1775.

32. J. P. Perdew, W. Burke and M. Ernzerhof, Phys. Rev. Lett., 1996, 77, 3865-3868.

10 33. J. Heyd, G. E. Scuseria and M. Ernzerhof, J. Chem. Phys., 2006, 124, 2204597.

34. A. V. Krukau, O. A. Vydrov, A. F. Izmaylov and G. E. Scuseria, J. Chem. Phys., 2006, 125, 224106.

35. A. Janotti, J. B. Varley, P. Rinke, N. Umezawa, G. Kresse and C. G. $15 \quad$ Van de Walle, Phys. Rev. B, 2010, 81, 085212.

36. T. Yamamoto and T. Ohno, Phys. Chem. Chem. Phys., 2012, 14, 589598.

37. J. K. Burdett, T. Hughbanks, G. J. Miller, J. W. Richardson and J. V. Smith, J. Am. Chem. Soc., 1987, 109, 3639-3646.

20 38. Y. Wang, H. W. Liu, H. M. Zhang, X. D. Yao and H. J. Zhao, Chem. Phys. Lett., 2011, 511, 82-86.

39. Y. Wang and G. S. Hwang, Surf. Sci., 2003, 542, 72-80.

40. Y. Wang, H. M. Zhang, Y. H. Han, P. R. Liu, X. D. Yao and H. J. Zhao, Chem. Comm., 2011, 47, 2829-2831.

25 41. A. Amtout and R. Leonelli, Phys. Rev. B, 1995, 51, 6842-6851.

42. H. Tang, H. Berger, P. E. Schmid and F. Levy, Solid State Commun., 1994, 92, 267-271.

43. H. Tang, H. Berger, P. E. Schmid, F. Levy and G. Burri, Solid State Commun., 1993, 87, 847-850.

30 44. Y. Wang, T. Sun, D. J. Yang, H. W. Liu, H. M. Zhang, X. D. Yao and H. J. Zhao, Phys. Chem. Chem. Phys., 2012, 14, 23332338.

45. F. Labat, P. Baranek, C. Domain, C. Minot and C. Adamo, J. Chem. Phys., 2007, 126, 12.

35 46. R. Hofmann, Solids and surfaces: A chemist's view of bonding in extended structures, VCH publishers, Inc., New York, 1988.

47. H. G. Yang, C. H. Sun, S. Z. Qiao, J. Zou, G. Liu, S. C. Smith, H. M. Cheng and G. Q. Lu, Nature, 2008, 453, 638-641.

48. H. M. Zhang, Y. Wang, P. R. Liu, Y. H. Han, X. D. Yao, J. Zou, H. M. Cheng and H. J. Zhao, ACS Appl. Mater. Interfaces, 2011, 3, 2472-2478. 